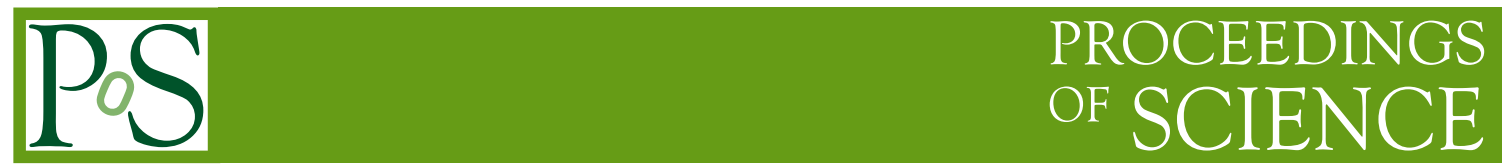

\title{
The top-quark gateway to new physics
}

\author{
Fabio Maltoni* \\ Centre for Cosmology, Particle Physics and Phenomenology \\ E-mail: fabio.maltoni@uclouvain.be
}

The top quark is the only fermion whose mass resides at the electroweak scale. Its role in the SM and in models of new physics together with its rich phenomenology provide a unique opportunity for exploring the $\mathrm{TeV}$ scale. In this talk I review the status of top-quark measurements after the Run I of the LHC and the exciting opportunities ahead in light of the possibility for the top quark to be a portal to new physics.

Frontiers of Fundamental Physics 14 - FFP14,

15-18 July 2014

Aix Marseille University (AMU) Saint-Charles Campus, Marseille

${ }^{*}$ Speaker. 